\title{
Publisher's Note: Candidate Source of Flux Noise in SQUIDs: Adsorbed Oxygen Molecules [Phys. Rev. Lett. 115, 077002 (2015)]
}

Hui Wang, Chuntai Shi, Jun Hu, Sungho Han, Clare C. Yu, and R. Q. Wu

(Received 23 November 2015; published 8 December 2015)

DOI: 10.1103/PhysRevLett.115.249901

PACS numbers: 85.25.Dq, 71.15.Mb, 73.50.Td, 74.25.Ha, 99.10.Fg

This paper was published online on 14 August 2015 with an omission of an author affiliation. Chuntai Shi's secondary affiliation should read as "Department of Physics, University of Wisconsin-Madison, Madison, Wisconsin 53706, USA." The corrected author and affiliation list should read as

Hui Wang, ${ }^{1,2}$ Chuntai Shi, ${ }^{2,3}$ Jun Hu, ${ }^{2}$ Sungho Han, ${ }^{2}$ Clare C. Yu, ${ }^{2}$ and R. Q. Wu ${ }^{1,2}$

${ }^{1}$ State Key Laboratory of Surface Physics and Department of Physics, Fudan University, Shanghai 200433, China

${ }^{2}$ Department of Physics and Astronomy, University of California, Irvine, California 92697-4575, USA

${ }^{3}$ Department of Physics, University of Wisconsin- Madison, Madison, Wisconsin 53706, USA

The affiliation list has been corrected as of 17 November 2015. The affiliation list is incorrect in the printed version of the journal. 